\title{
Comment la Révolution abolit la dignité de
} maréchal de France

How the Revolution abolished the dignity of the marechal of France

\section{Fadi El Hage}

\section{(2) OpenEdition \\ 1 Journals}

Édition électronique

URL : https://journals.openedition.org/ahrf/10877

DOI : 10.4000/ahrf.10877

ISSN : 1952-403X

Éditeur :

Armand Colin, Société des études robespierristes

Édition imprimée

Date de publication : 1 décembre 2008

Pagination : $51-75$

ISSN : 0003-4436

Référence électronique

Fadi El Hage, «Comment la Révolution abolit la dignité de maréchal de France », Annales historiques de la Révolution française [En ligne], 354 | octobre-décembre 2008, mis en ligne le 01 décembre 2011, consulté le 22 avril 2022. URL : http://journals.openedition.org/ahrf/10877 ; DOI : https://doi.org/ 10.4000/ahrf. 10877 


\title{
COMMENT LA RÉVOLUTION ABOLIT LA DIGNITÉ DE MARÉCHAL DE FRANCE
}

\author{
Fadi EL HAGE
}

\begin{abstract}
En 1793 fut aboli le titre de maréchal de France. S'il survécut quatre ans aux profondes transformations de la société et de l'armée sous la Révolution, c'est parce que, au temps de la monarchie constitutionnelle, l'Assemblée tenta de réformer cet éminent titre pour en faire le plus haut grade militaire. En s'inspirant des critiques portées à l'encontre du maréchalat et du commandement des armées en général, et en agissant parfois selon les circonstances, les députés essayèrent de redonner à ce titre prestigieux un crédit qui paraissait avoir été entamé par les abus de la monarchie d'Ancien Régime. L'échec des maréchaux de Rochambeau et de Luckner mit un terme à ces entreprises. La dignité « rétablie » en 1804 n'était pas exactement similaire, bien que reprenant certaines traditions d'Ancien Régime en même temps que certaines réformes révolutionnaires.
\end{abstract}

Mots-clés : maréchaux, commandement, bâton, mérite, contrerévolution.

Le 21 février 1793 fut présenté à la Convention un décret « relatif à l'organisation de l'armée et aux pensions de retraite et traitements de tout militaire, de quelque grade qu'il soit $\rangle^{1}$. L'article 5 stipulait que « toutes les dénominations de lieutenant-colonel, colonel, maréchal-decamp, lieutenant-général et de maréchal de France, sont supprimées $»^{2}$.

En une seule phrase, le maréchalat de France était aboli, après une existence attestée depuis Philippe Auguste jusqu'aux quatre premières

(1) Archives parlementaires de 1787 à 1860, Paris, Dupont, 1901, LIX, p. 163.

(2) Ibid., p. 164. 
années de la Révolution. Le fait qu'il ait survécu à l'Ancien Régime peut paraître surprenant, car il en constituait l'une des dignités les plus éminentes. En effet, en tant que grands officiers de la couronne, les maréchaux étaient très impliqués dans la vie politique et militaire du royaume. Sous le règne de Louis XVI, trois maréchaux furent ministres (ou plutôt trois ministres furent faits maréchaux), sans compter Broglie, nommé ministre de la Guerre le 11 juillet 1789. Plusieurs d'entre eux assistèrent à l'Assemblée des notables de 1787 et contribuèrent au maintien de l'ordre public, à l'exemple des maréchaux de Biron à Paris lors de la guerre des Farines en 1775, et de Vaux à Grenoble après la journée des Tuiles en 1788. Les détenteurs de la dignité veillèrent ainsi au respect de leurs intérêts sociaux mais aussi à celui de l'ordre établi par le roi, à qui ils avaient juré fidélité dans leur serment de maréchal ${ }^{3}$.

En dépit de son passé intimement lié à la monarchie, le maréchalat fut ménagé, quitte à être transformé quelque peu afin de le rendre plus viable en Révolution. Ces transformations n'étaient pas issues d'idées tout à fait neuves, encore moins improvisées, car le XVIII siècle fut un siècle de réflexions sur cette institution, son utilité et son organisation, tant du côté de l'État que de celui de l'opinion publique. La gestion de la dignité sous la monarchie absolue suscita des critiques qui aboutirent, en partie du moins, à sa réforme.

Les modifications opérées sous la monarchie constitutionnelle causèrent une rupture par rapport à la situation précédente, ce qui permit le maintien du titre de maréchal pendant quatre ans, dans une France ayant aboli les privilèges et s'opposant irrévocablement au roi, après une brève tentative de conciliation.

Une décennie sépare l'abolition du maréchalat et l'instauration, le 18 mai 1804, des maréchaux d'Empire. Jacques Godechot considérait

(3) Le serment de maréchal de France resta inchangé de la seconde moitié du XVII siècle à la fin de l'Ancien Régime. Curieusement, une retranscription de sa formule est conservée dans le dossier militaire de Rochambeau (SHD, 2Yd 256), alors qu'il ne fut pas prêté sous la Révolution, puisqu'il n'y avait logiquement aucune référence à la Nation : « Vous jurez et promettez à Dieu de bien et fidèlement servir le Roy envers et contre tous sans en excepter personne, en l'office de maréchal de France dont Sa Majesté vous a pourvu. De n'avoir intelligence avec qui que ce soit au prejudice de l'Estat et de son service ; de luy reveler tout ce que vous entendrez luy estre prejudiciable ; de ne recevoir pension d'autre Prince que de Sa Majesté ; de faire vivre en bon ordre, justice et police les gens de guerre qui sont et seront à sa solde ; que vous les empescherez de fouler et oppresser son peuple et leur ferez soigneusement garder les Ordonnances ; que de votre part vous garderés et entretiendrés lesdites ordonnances en tout ce qui vous sera ordonné, et ferez en ce qui concerne ledit office de marechal de France tout ce qu'un bon et fidele sujet doit et est tenu de faire ; et pour mieux exécuter ce que dessus, Sa Majesté vous fait mettre en main le bâton de marechal de France ainsy qu'il a esté fait à vos prédécesseurs ». 
que ceux-ci n'incarnaient que le rétablissement d'une institution d'Ancien Régime, marquant « dans l'armée, la tendance générale du régime napoléonien vers le retour à une société très hiérarchisée et conservatrice $»^{4}$. Il s'agit ici de l'opinion d'un historien qui percevait le Premier Empire comme plus proche de la monarchie de Louis XIV que de la Révolution ${ }^{5}$. Or, il était inconcevable que l'empereur négligeât l'héritage révolutionnaire, car il ne pouvait, et ne voulait, en aucun cas rétablir l'Ancien Régime. Le maréchalat d'Empire tenait-il plus du maréchalat d'Ancien Régime, qu'il n'était imprégné des réformes menées par l'Assemblée constituante?

\section{La dignité de maréchal de France face à l'opinion publique du siècle des Lumières}

À la fin du XVIII ${ }^{e}$ siècle, le maréchalat était perçu comme une dignité sur le déclin. Des questions se posaient alors sur les activités de ses titulaires, qui paraissaient en inadéquation avec l'imaginaire dominant dans l'opinion publique. En se demandant si « tous les maréchaux de France aujourd'hui ont gagné des batailles ", le père de Mirabeau présentait avant tout leur dignité comme fortement liée au commandement des armées $^{6}$. Cette fonction semblait aller de soi dans les esprits, ce qui explique la généralisation de l'expression « grade de maréchal de France» bien avant la Révolution?

Le maréchalat ne se résumait pourtant pas au commandement. Le temps de paix ne nécessitait pas la formation d'armées à mener en campagne. D'autres activités occupaient les maréchaux, comme les gouvernements de province, ou le tribunal du point d'honneur, qui était la seule grande institution propre à ces derniers ${ }^{8}$. Sa réunion était l'un des rares

(4) Jacques Godechot, Les Institutions de la France sous la Révolution et l'Empire, Paris, PUF, Dito, 1998, p. 606.

(5) Les Constitutions de la France depuis 1789, Paris, Flammarion, 1995, p. 184.

(6) Victor Riqueti de Mirabeau, L'ami des hommes ou Traité de la population, Avignon, 1756, p. 182.

(7) Le maréchal de Vauban considérait notamment que le commandement incombait naturellement aux maréchaux de France (Albert Rochas D’AygLun, Vauban : sa famille et ses écrits : ses oisivetés et sa correspondance : analyse et extraits, Paris, Berger-Levrault, 1910, II, p. 646). L'expression « grade de maréchal de France » apparut au moins à partir du XVII siècle.

(8) En vertu du règlement du 18 mars 1776 , les gouvernements de province de première classe (il y en avait deux) étaient attribués uniquement aux princes du sang et aux maréchaux de France. Sur le tribunal des maréchaux de France, nous renvoyons à Pascal BrioIst, Hervé Drevillon, Pierre SERnA, Croiser le fer. Violence et culture de l'épée dans la France moderne (XVI $-X V I I I^{e}$ siècle), Seyssel, Champ Vallon, Epoques, 2002, p. 349-362. 
moments durant lesquels ils se rassemblaient chez leur doyen. La maréchale de Beauvau confessa que « le plus grand mal [qu'ait...] fait la révolution [à son mari...] a été de le priver du noble plaisir qu'il trouvoit à être encore, dans l'âge avancé, utile à la chose publique ; comme gouverneur de province, comme membre du tribunal des maréchaux de France $»^{9}$. Ce tribunal de la noblesse ne pouvait aucunement trouver sa place dans une France ayant aboli les privilèges, d'autant plus qu'il était peu apprécié, y compris par les aristocrates. Dans ses mémoires, le comte de Tilly s'attacha ainsi à le dénigrer systématiquement, en remettant en cause la légitimité et le mérite de ses juges.

Cette question du mérite, déjà ancienne, se fit plus insistante, si bien que son évocation était lourde de sens parmi les députés de la Constituante. Le 4 mars 1791, avec son emphase habituelle, Mirabeau évoqua les travers des promotions de maréchaux de France sous l'Ancien Régime, qui étaient, selon lui, au nombre de deux : « un bâton de maréchal de France donné pour un assassinat [...] les bâtons de maréchaux de France donnés pour des assiduités d'antichambre, à un courtisan $»^{10}$.

Il est évident que le tribun pensait aux exemples de Vitry et de Soubise. Le maréchal de Vitry fut nommé le 24 avril 1617 pour avoir, le jour même, arrêté et fait assassiner Concini à la demande du jeune Louis XIII. Il fut ainsi récompensé pour avoir accompli une action politique sanglante, permettant au roi de se défaire d'un homme trop puissant. Quant au prince de Soubise, il était, pour l'auditoire de Mirabeau, l'incarnation du courtisan intime du roi récompensé pour son assiduité et sa complaisance à Versailles. Son cas était suffisamment récent pour être connu de tous. Protégé de Madame de Pompadour, il fut vaincu à Rossbach le 5 novembre 1757. L'année suivante, il remporta la bataille de Lutzelberg (10 octobre 1758), ce qui lui valut le bâton. L'avocat parlementaire Barbier la relata dans son Journal, d'après ce qui en avait été dit à Paris, si bien qu'il refusa de lui en attribuer la victoire. Elle fut portée au crédit de Chever ${ }^{11}$. Orphelin, ce dernier servit très jeune dans l'armée et s'éleva jusqu'au grade de lieutenant-général. On explique souvent qu'il n'accéda

(9) Marie-Charlotte de Beauvau, Souvenirs de la Maréchale princesse de Beauvau, Paris, Techener, 1872, p. 5.

(10) Archives parlementaires de 1787 à 1860, op. cit., 1886, XXIII, p. 662.

(11) Edmond-Jean-François BARBIER, Chronique de la Régence et du règne de Louis XV (1718-1763) ou Journal de Barbier, Paris, Charpentier, 1857, VII, p. 101 ; Jean Chagniot, Paris et l'armée au XVIII siècle, Paris, Economica, 1985, p. 534. 
jamais au maréchalat parce qu'il n'était pas d'origine noble ${ }^{12}$. Un tel destin fit de lui une figure appréciée du tiers état. L'opuscule Apparition du général Chevert, au tiers état, rédigé après la décision du roi de doubler le nombre de députés du Tiers, fut conçu dans cette optique ${ }^{13}$. Il met en valeur le mérite de Chevert qui ne fut pas récompensé, contrairement à Fabert, souvent considéré à tort comme roturier. Maréchal nommé par Mazarin en 1658, ce dernier était un exemple de «l'idéal incarné » défini par Hervé Drévillon, à savoir un modèle « de sagesse et de modération [...] exemple d'obéissance et d'exactitude au service $\gg^{14}$. À l'inverse, il paraissait inconcevable qu'un noble aussi récent s'élevât uniquement par son mérite. Omettant le fait qu'il était une « créature » de Mazarin, comme l'écrivit Henri de Campion, des causes surnaturelles furent évoquées, et notamment un pacte avec le Diable ${ }^{15}$ :

« Parmi tant d'exemples que je pourrois citer, je me contenterai de vous rappeler celui du Maréchal Fabert, qui, comme moi \& semblable à vous, Tiers État, n'eut pour recommandation que son mérite \& sa bravoure. Cependant il s'éleva jusqu'au faite des honneurs, \& mourut au plus haut degré de grandeur. On osa avancer, dans ces temps où la France étoit encore enveloppée des ténèbres épaisses de la superstition, qu'il avoit fait pacte avec le Diable, auquel il s'étoit donné tout entier, \& qu'en récompense l'Esprit malin l'avoit toujours accompagné, conduit, guidé \& fait réussir dans tous ses vaillans exploits. De là on forgea cette fable, aussi incroyable que ridicule, par laquelle on supposa qu'il étoit mort d'une manière tragique, en soutenant affirmativement que ce même Diable étoit venu l'étrangler $»^{16}$.

Catinat, promu en 1693, était l'autre maréchal « roturier » (il ne l'était pas plus que Fabert) représentant « l'idéal incarné ». Sa réputation de victime de la cour et de philosophe lui donna une popularité posthume au siècle des Lumières. L'apothéose de la gloire de Catinat eut lieu en 1775 , avec le concours organisé par l'Académie française, consistant à rédiger un éloge de ce maréchal ${ }^{17}$.

(12) L'épitaphe de Chevert en l'église Saint-Eustache à Paris, est fort connue : « Il s'éleva, malgré l'envie, à force de mérite, et chaque grade fut le prix d'une action d'éclat. Le titre seul de maréchal de France a manqué, non pas à sa gloire, mais à l'exemple de ceux qui le prendront pour modèle ».

(13) «Le Roi vous appelle à ses États Généraux, en nombre égal aux deux autres Ordres » (Apparition du général Chevert, au Tiers État, [1789 ?], p. 15).

(14) Hervé Drevillon, L'Impôt du sang, Paris, Tallandier, 2005, p. 344.

(15) Ces rumeurs provenaient vraisemblablement des milieux aristocratiques.

(16) Apparition du Général Chevert, au Tiers État [1789 ?], p. 11.

(17) Ce concours, auquel le tacticien Guibert participa, fut remporté par La Harpe. 
Cette exclusion d'une certaine catégorie d'officiers fut sentie d'une manière d'autant plus forte que l'accès à la dignité paraissait se refermer de plus en plus. La fournée de 1775 fut remarquable à ce niveau, car, sur les sept promus, cinq étaient fils ou petits-fils de maréchaux ${ }^{18}$. Ils étaient de surcroît déjà ducs. Le phénomène n'était pas inédit, mais il n'avait jamais été aussi important ${ }^{19}$. Le maréchalat avait été une véritable promotion sociale pour un marquis ou un simple chevalier. Ainsi, il avait contribué à l'accession du marquis de Villars aux titres de duc et pair, pour prendre un exemple significatif. En revanche, pour quelqu'un qui avait déjà ce rang, la dignité n'était pas un moyen d'ascension, mais un ornement supplémentaire.

Les promotions de maréchaux de France se faisaient le plus souvent par fournées, y compris en temps de paix. Dans ses Mémoires pour l'instruction du Dauphin, Louis XIV avait expliqué la large promotion de chevaliers du Saint-Esprit de 1661 par l'absence d'attribution de l'ordre depuis 1633, ainsi que par l'idée que « nulle récompense ne coûte moins à nos peuples $[\ldots]$ que ces distinctions de rang $»^{20}$. Nous pouvons considérer les fournées de maréchaux de la même manière. Elles coûtaient moins au Trésor royal que d'autres charges ou fonctions qui étaient également susceptibles d'être attribuées.

Les fournées posaient-elles un problème financier à l'État? Au vu du montant des appointements de maréchal de France (13 522 livres et 10 sous), ce n'étaient pas les revenus de la dignité qui coûtaient le plus au Trésor, mais les pensions connexes ${ }^{21}$. La rente de 30000 livres attribuée

(18) Il est à noter que ces maréchaux appartenaient à des familles comptant parmi les plus importantes du royaume. Duras descendait des maréchaux de 1675 et 1741 du même nom, le premier étant par ailleurs neveu de Turenne. Les frères Noailles et Mouchy étaient les petits-fils et fils des maréchaux de Noailles de 1693 et 1734 ; c'était aussi la première (et la seule, en fait) fois que deux frères faisaient partie de la même fournée. Le maréchal d'Harcourt de 1775 était, en plus d'être le descendant du maréchal de 1283, le frère de celui de 1746 et le fils du maréchal de 1703. Quant au duc de Fitz-James, il n'était autre que le fils du maréchal de Berwick, fils naturel de Jacques II.

(19) Dans une promotion au nombre similaire, comme celle de 1693, seuls deux nommés étaient déjà ducs et pairs, Villeroy et Noailles. La promotion de 1724, la première en temps de paix est caractérisée par un nombre important de fils et petits-fils de maréchaux, et ne comportait que trois ducs et pairs.

(20) Mémoires et lettres de Louis XIV, Paris, Plon, Les Cahiers de l'Unité Française, 1942, p. 33.

(21) Dans le cas du maréchal de Ségur, les appointements de maréchal de France ne comptaient que pour $17,2 \%$ de ses revenus ordinaires (nous ne prenons pas en compte les sommes extraordinaires qu'il reçut). Cette proportion est inférieure à celle que l'on peut mesurer avec les revenus du duc de Croÿ $(22,3 \%$ ) ou du maréchal d'Aubeterre (36\%), mais supérieure à celle calculée sur les revenus de Vaux (13,5\%). Contrairement à Croÿ et Aubeterre, Ségur avait une pension sur le Trésor royal de 13000 livres, soit presque autant que les appointements de maréchal, tandis que Vaux bénéficiait d'un traitement sur l'extraordinaire des guerres d'un montant de 36000 livres, qui était presque trois fois supérieur à ces mêmes appointements. 
aux maréchaux de France en 1791, ne paraît faible que si elle est perçue comme une compensation de la suppression des revenus supplémentaires qui n'avaient rien à voir avec la dignité de maréchal ${ }^{22}$.

L'augmentation des effectifs commença au $\mathrm{XVI}^{\mathrm{e}}$ siècle, afin de relever temporairement l'effectif ordinaire de quatre maréchaux. Les différents événements survenus dans le royaume de France contribuèrent à la hausse de leur nombre. Il était nécessaire au début de la monarchie des Bourbons de rallier les mécontents. Ensuite, l'État voulut de surcroît récompenser de longues carrières au service du roi ou des coups d'éclat comme celui de Villars à Fredlingen en 1702. La fin du règne de Louis XIV vit un doublement des effectifs, afin de répondre aux impératifs de la guerre et de prévenir les baisses trop importantes du nombre des maréchaux.

Les circonstances de ces «fournées de maréchaux de France » furent sujettes à caution. Elles servaient souvent à occulter le fait qu'un ou deux individus en particulier étaient spécialement destinés à être promus. Le baron de Besenval présenta dans ses mémoires la fournée de 1783 comme une manœuvre politique. Alors que Ségur et Castries, respectivement ministres de la Guerre et de la Marine, étaient contestés par une partie de la cour, il proposa une solution pour montrer qu'ils avaient toujours la faveur du roi : que celui-ci les fit maréchaux de France. C'est effectivement ce qui se passa. Pour masquer la manœuvre contestable, expliquée tant bien que mal par le mérite des deux hommes dans la conduite de la guerre d'Amérique, on en nomma d'autres en même temps ${ }^{23}$.

La hausse des effectifs qui eut lieu au XVIII ${ }^{e}$ siècle atteignit des proportions inédites. On pouvait compter vingt maréchaux de France en 1703, ainsi que de 1758 à 1761 . On se rapprocha de ce chiffre en 1784 avec dix-neuf maréchaux, ce qui peut paraître surprenant, car la France était alors en paix. Les effectifs étaient fort élevés, plus que de raison. La rareté des places n'était plus de mise, ce qui réduisit l'intérêt de la dignité au sein de l'opinion. En revanche, elles l'étaient de plus en plus pour les familles nobles récentes, exclues d'un titre méritoire devenu l'apanage

(22) Dans une même lettre envoyée en février 1793 au ministre de la Guerre Beurnonville, puis en octobre de la même année à son successeur Bouchotte, Ségur rappelait « ces services et ces blessures [le maréchal avait notamment perdu un bras à la bataille de Lawfeld en 1747] [qui lui] avaient fait obtenir cent vingt-huit mille livres de traitement [que] l'Assemblée [avait réduits] à trente mille livres » (SHD, 2 Yd 250).

(23) Pierre de Besenval, Mémoires du Baron de Besenval, Paris, Baudoin frères, 1828, II, p. $139-148$. 
des aristocrates, du moins des familles déjà intégrées dans le " circuit » du maréchalat. Cette évidence influença grandement les réflexions menées avant et au début de la Révolution.

\section{Les maréchaux dans la tourmente révolutionnaire : de l'Ancien Régime à l'émigration}

La convocation des États généraux donna un aperçu de l'opinion portée sur la dignité de maréchal de France, par l'intermédiaire des cahiers de doléances. Le maréchalat ne semble pas avoir été la préoccupation première du tiers état, plus sensible aux difficultés de la vie quotidienne. Aussi est-il difficile de trouver des demandes explicites pour un accès du tiers état au bâton de maréchal. Sur le plan militaire, le Tiers réclamait surtout l'accès aux grades militaires pour ses membres, sans trop insister sur cette haute dignité ${ }^{4}$. Le clergé n'était pas beaucoup plus prolixe. À Rodez, il demanda juste à ce que le maréchalat fût le seul moyen de conférer la noblesse héréditaire ${ }^{25}$.

Les cahiers de la noblesse étaient logiquement plus explicites à propos de cette dignité. Une grande place était accordée au tribunal des maréchaux, pour lequel on ne réclamait pas tant sa suppression que l'interdiction d'emprisonner des gentilshommes par simples lettres de cachet ou la réduction des champs de compétence de sa juridiction ${ }^{26}$. L'idée d'une volonté de réformer la dignité émergeait ; ainsi, le cahier de la noblesse de la banlieue de Paris demandait à ce « que le pouvoir très-précieux des maréchaux de France soit circonscrit dans ses limites nouvelles $\gg{ }^{27}$. Il s'agissait d'établir un conseil militaire national, « composé de maréchaux de France \& d'officiers de tous grades $»^{28}$. Ils devaient rester cantonnés à des fonctions militaires.

Le cahier de la noblesse de Cézanne et Châtillon-sur-Marne, lieux pourtant très éloignés, a priori, des enjeux politiques et militaires du pays,

(24) C'est l'idée que l'on peut avoir à la lecture du troisième volume du Résumé général, ou Extrait des cahiers de pouvoirs, instructions, demandes \& doléances, remis par les divers bailliages, sénéchaussées \& pays d'États du royaume, à leurs députés à l'Assemblée des États-généraux, ouverts à Versailles le 4 mai 1789, mais rappelons que cet ouvrage ne regroupe pas la totalité des revendications des différents ordres, loin s'en faut.

(25) Ibid., I, p. 310. Nous pouvons aussi penser que les cahiers de Troyes, Mantes et Meulan incluaient le maréchalat parmi les grades militaires pour lesquels ils réclamaient l'égalité d'accès (ibid., I, p. 311).

(26) Ibid., II, p. 335-336.

(27) Ibid., II, p. 335.

(28) Ibid., II, p. 332. 
va plus loin en demandant clairement à ce « qu'on ne multiplie pas au-delà du besoin, \& par faveur, le nombre de maréchaux de France, au détriment de cette éminente dignité, \& à la surcharge du royaume, par les traitemens qu'il faut leur faire pour en soutenir l'éclat $»^{29}$. Cette revendication est probablement la plus intéressante, car elle souligne l'idée que les fournées de maréchaux avilissaient la dignité, comme cela avait été dit un siècle auparavant à propos de la «monnaie de Turenne $»^{30}$. La demande de ne pas multiplier les maréchaux est à associer à celle du cahier du Vermandois voulant qu'on n'éclipse pas le mérite avec une fortune ou un grand nom, comme cela avait pu être le cas en 1775 avec une promotion faible en homines novi ${ }^{31}$.

À la veille de la Révolution, le maréchalat n'était pas remis en cause dans son existence, mais une réforme institutionnelle était souhaitée, afin de limiter les effectifs, surtout pour des raisons de prestige. À l'instar de la monarchie, la dignité de maréchal de France n'était pas appelée à être supprimée.

Les premières difficultés apparurent lors des événements de juillet 1789. Les troupes rassemblées à Versailles le 11 furent confiées au nouveau ministre de la Guerre, le maréchal de Broglie, dont le premier souci était d'éviter toute effusion de sang ${ }^{32}$. Le député du Tiers, Pellerin, nota qu'une rumeur voulait qu'il devînt maréchal-général des camps et armées du roi, charge qui aurait dû lui donner une réputation analogue à celle de Turenne, Villars, ou Maurice de Saxe, pour ne citer que les derniers titulaires de cette charge ${ }^{33}$. Il n'y eut pas de mouvement de troupes sur Paris, la Bastille fut prise, et Broglie partit le lendemain vers son gouvernement des Trois-Évêchés et d'Alsace, où il fut pris à partie, alors qu'il avait connu jadis la faveur de l'opinion publique. Une rumeur circula même selon laquelle il aurait été décapité34. Il n'en fut rien. Même si Louis XVI fit rédiger en sa faveur le 16 juillet des patentes de maréchal-général lui

(29) Ibid., II, p. 337.

(30) Trois jours après la mort de Turenne près de Salzbach le 27 juillet 1675, Louis XIV nomma d'un seul coup huit maréchaux. Madame de Cornuel avait alors affirmé que «le Roi a changé son louis d'or en louis de cinq sous ».

(31) Résumé général..., op. cit., II, p. 323.

(32) Pierre CARON, «La tentative de contre-révolution de juin-juillet 1789 », Revue d'histoire moderne, VIII, 1906-1907, p. 5-34 et 649-678.

(33) Joseph-Michel Pellerin, Correspondance inédite de J.-M. Pellerin, député du Tiers-État de la sénéchaussée de Guérande, Paris, Sauton, 1883, p. 89.

(34) Une rumeur similaire circula concernant le maréchal de Mailly (Martin, Voyage à Paris en 1789 de Martin, faiseur de bas d'Avignon, Avignon, Roumanille, 1890, p. 46). 
permettant de commander les maréchaux de France, il préféra partir à l'étranger ${ }^{35}$.

Castries le suivit rapidement sur le chemin de l'exil. Ils jouèrent un rôle de premier ordre parmi les émigrés, notamment au conseil formé autour du frère du roi, ainsi qu'à l'éphémère armée d'émigrés. Tous deux moururent en exil, Castries en 1800, Broglie en 1804. La question de leur maintien dans leur titre se posa à l'Assemblée constituante. Le 5 mars 1791, Victor de Broglie y défendit la cause de son père, en affirmant qu'on avait persuadé celui-ci qu'il y avait une situation de « danger ». S'il n'était pas revenu en France, comme cela était réclamé par l'Assemblée, c'est parce que sa mauvaise santé l'en aurait empêché ${ }^{36}$. Malgré le désaveu du maréchal, son fils lui permit de conserver son titre, mais cela s'avéra impossible après $1792^{37}$. Broglie et Castries se dirent toujours maréchaux, puisqu'ils ne reconnaissaient pas la monarchie constitutionnelle, encore moins la République. La fidélité de ces deux maréchaux à la monarchie d'Ancien Régime montrait que le maréchalat était trop lié à l'ancien ordre, et qu'il devait être réformé.

\section{La régénération du maréchalat}

L'abolition des privilèges du 4 août 1789 impliquait que la noblesse n'existerait plus en tant qu'ordre privilégié. Les juridictions spéciales étaient donc appelées à disparaître. Ainsi fut aboli le tribunal des maréchaux de France le 7 septembre $1790^{38}$. Au même moment, la régénération administrative de la France priva ceux-ci d'une importante source de revenus, du fait de la suppression des provinces au profit de la départementalisation. Si le maréchalat était indirectement touché par ces mesures, il n'allait pas tarder à être lui-même perçu comme une institution à révolutionner.

(35) Les lettres de maréchal-général de Broglie peuvent être trouvées dans Charles-Auguste D'Allonville, Mémoires secrets de 1770 à 1830, Paris, Werdet, 1838, II, p. 166-167. Seul Villars, jusqu'alors, s'était vu notifier explicitement dans ses provisions de maréchal-général des camps et armées du roi qu'il commandait les maréchaux de France. À la date du 16 juillet 1789, Contades était l'unique maréchal plus ancien que Broglie, ce qui signifie que ce dernier pouvait déjà commander les autres maréchaux.

(36) Archives parlementaires de 1787 à 1860, op. cit., 1886, XXIII, p. 667-668.

(37) Les maréchaux de Broglie et de Castries ont disparu de la liste des maréchaux de France à la page 5 de l'État militaire de la France pour l'année 1793.

(38) Gabriel Le Barrois D’Orgeval, Le Maréchalat du Moyen Âge à nous jours : tome II Nouveau Régime, Paris, Occitania, 1932, II, p. 19. 
Le 4 mars 1791 eut lieu le premier débat important sur la dignité, qui n'était désormais plus que le grade le plus élevé de l'armée de terre, au même titre que celui d'amiral pour la marine. Il était désormais théoriquement accessible à tous les hommes, mais pas aux femmes, en dépit d'une requête déposée à l'Assemblée nationale ${ }^{39}$. La discussion porta essentiellement sur les effectifs à maintenir, ainsi que sur le traitement à attribuer aux titulaires ${ }^{40}$. Il fut décidé qu'il n'y aurait que six maréchaux de France en activité. Les objections concernèrent généralement le temps de paix, qui ne nécessitait pas théoriquement la nomination de maréchaux. Or, une interruption des promotions aurait été injuste pour ceux qui avaient bien servi la Nation, le député d'André citant les noms de Rochambeau et de Bouillé, qui jouèrent un rôle majeur dans le maintien de l'ordre dans l'Est, en particulier le deuxième à Nancy. À l'opposé, le député d'Estourmel affirma qu'il fallait plus de six maréchaux en temps de guerre, prenant pour exemple la «monnaie de Turenne ». Les effectifs de 1791 étant bien supérieurs, certains titulaires furent mis à la retraite, tout en conservant leur traitement ${ }^{41}$. Celui-ci posa problème quant à son montant, car il différait jadis selon les titulaires, qui cumulaient souvent différentes charges. Il fut finalement fixé à 30000 livres pour les maréchaux d'active.

La tentative de fuite des 20 et 21 juin 1791 fut la dernière occasion pour Louis XVI de tenter de reprendre en main son autorité en tant que roi. Aussi était-il prévu, dès que la frontière serait atteinte, de faire Bouillé maréchal de France ${ }^{42}$. L'échec de cette entreprise provoqua un divorce irréversible entre le roi et la Nation, mais il fallait sauver les apparences pour sauver la nouvelle constitution. Les députés restituèrent donc à Louis XVI ses pouvoirs dès le mois de juillet. Le 14 septembre, celui-ci accepta la constitution. Le roi des Français n'allait pas tarder à découvrir les

(39) Dans une Requête des Dames, à l'Assemblée nationale, nous trouvons un curieux projet de décret remis aux députés demandant la promotion de femmes aux « emplois, récompenses \& dignités militaires », ainsi qu'une attribution alternée du bâton de maréchal entre hommes et femmes, cette dernière revendication étant sûrement teintée d'ironie : «n'en exceptons pas même le bâton de maréchal de France ; \& pour que justice puisse être également faite, nous ordonnons que cet instrument si utile passera alternativement entre les mains des hommes \& des femmes » (Requête des Dames, à l'Assemblée nationale, 1789, p. 13). Nous remercions monsieur Pierre Serna de nous avoir fait découvrir ce document.

(40) Archives parlementaires de 1787 à 1860, op. cit., 1886, XXIII, p. 662-663.

(41) Le 24 novembre 1791, lors d'une séance de l'Assemblée législative, les six maréchaux d'active désignés par le roi furent énumérés : Contades, Mouchy, Mailly, Beauvau, Laval, Ségur (Archives parlementaires de 1787 à 1860, op. cit., 1890, XXXV, p. 349).

(42) Claude-Antoine-Gabriel De Cholseul, Relation du départ de Louis XVI, le 20 juin 1791, Paris, Baudoin frères, 1822, p. 53. 
limites de son pouvoir, aussi bien en vertu de la constitution qu'à cause de l'accaparement des prérogatives de l'exécutif par l'Assemblée législative.

La constitution de 1791 réglementait la nomination des maréchaux de France par le roi. L'article 2 du chapitre IV souligne que c'est le roi qui conférait « le commandement des armées et des flottes, et les grades de maréchal de France et d'amiral $»^{43}$. Cependant, le pouvoir régalien en ce domaine était fortement nuancé à l'article premier du chapitre III de la constitution, qui attribuait à l'Assemblée législative le pouvoir « de statuer annuellement, après la proposition du roi [...] sur la solde et le nombre d'individus de chaque grade $»^{44}$. Louis XVI n'avait désormais pour rôle que de proposer des noms de nouveaux maréchaux - dans la limite fixée par l'Assemblée, sauf dérogation - aux députés qui avaient le loisir de les valider ou pas. Avant 1791, c'était le ministre de la Guerre qui proposait des noms, inscrits sur une feuille, au roi. Le souverain rayait ensuite les noms de ceux qu'il écartait. Louis XVI avait perdu le monopole de la nomination des maréchaux de France.

Le rôle du roi, tel qu'il était défini dans la constitution, fut rapidement mis en difficulté, a fortiori dans le cas des nominations de maréchaux de France. La guerre semblait imminente, alors que l'armée française était fragilisée par l'émigration. À part Ségur, les maréchaux étaient tous au moins septuagénaires ; ils étaient trop infirmes pour commander. Il fallait nommer de nouveaux maréchaux. C'est pour cela que, le 14 décembre 1791, Narbonne, ministre de la Guerre, demanda la formation de trois armées, qu'il fallait attribuer à Rochambeau, Luckner et La Fayette. Afin de donner plus de poids à ces nominations, il demanda l'attribution du bâton de maréchal aux deux premiers, en vertu d'un élargissement exceptionnel du nombre maximum de maréchaux imposé depuis peu : «Sa Majesté eût désiré que l'organisation militaire lui permît de donner le grade de Maréchal de France à MM. de Rochambeau et Luckner $»^{45}$. La phrase paraît en parfaite conformité avec la constitution de 1791. Rochambeau conserva dans ses Mémoires cette version des faits, en notant que « le roi proposa à l'Assemblée nationale une dérogation au décret de

(43) Les Constitutions de la France depuis 1789, op. cit., p. 55.

(44) Ibid., p. 50.

(45) Journal militaire, année 3, 1792, partie I, p. 37. Louis XVI aurait refusé de nommer la Fayette maréchal, même si Narbonne lui avait répondu qu'il y serait obligé par la pression populaire. Il aurait été cependant curieux de voir la Fayette promu à ce grade à trente-quatre ans. En effet, il était lieutenant-général depuis une période trop récente, et nul n'était devenu maréchal à la trentaine depuis la régence d'Anne d'Autriche. 
l'Assemblée constituante, qui avait fixé à six le nombre de maréchaux de France, et une augmentation de ce nombre en faveur de M. de Luckner et de [lui] $\gg^{46}$. Or, il semble que l'initiative était due à Narbonne, ce qui se confirme à la lecture d'une lettre de Madame Élisabeth datant du 14 décembre même : «M. de Narbonne a parlé ensuite pour dire que le général Rochambeau et Luckner allaient être maréchal [sic] de France $»^{47}$.

Pourquoi avoir choisi Rochambeau et Luckner? La principale explication serait la faveur dont ils jouissaient auprès de l'Assemblée. Ils avaient juré fidélité à la Constituante plusieurs fois, y compris au moment de la fuite avortée de Louis XVI. Leurs actions passées étaient plus susceptibles d'attirer l'attention des députés que celle de la famille royale. C'est pour cela que, dès le 20 septembre 1791, Barnave avait suggéré à Marie-Antoinette que le roi devait distinguer, au moins par des mots, ces deux généraux ${ }^{48}$.

Jean-Baptiste Vimeur de Rochambeau naquit en 1725. Il s'était distingué lors des guerres de Succession d'Autriche et de Sept Ans, mais le sommet de sa carrière eut lieu en 1780, quand il prit la tête du corps expéditionnaire d'Amérique. Il incarna rapidement, à l'instar de La Fayette, les « idées nouvelles $»^{49}$.

Nicolas Luckner était, quant à lui, Bavarois. Né en 1722, il servit plusieurs souverains et combattit notamment à Rossbach. En échange d'une forte somme d'argent et du grade de lieutenant-général, il accepta de passer au service de la France en 1763. Il incarnait non seulement l'étranger qui préféra la France aux monarchies despotiques européennes, mais le «modèle militaire prussien », que la France tentait depuis la guerre de Sept Ans d'assimiler ${ }^{50}$.

Si Narbonne respectait la constitution en proposant au nom du roi la nomination des deux nouveaux maréchaux, il est indéniable que le rôle

(46) Jean-Baptiste-Donatien de Vimeur de Rochambeau, Mémoires militaires, historiques et politiques de Rochambeau, ancien maréchal de France, Paris, Pillet aîné, 1824, I, p. 391.

(47) Correspondance de madame Élisabeth de France, sœur de Louis XVI, Paris, Plon, 1868, p. 374.

(48) Marie-Antoinette Correspondance (1770-1793), Paris, Tallandier, 2005, p. 616.

(49) Mathieu Dumas, rapporteur du comité militaire de l'Assemblée, souligna par deux fois le rôle de Rochambeau en Amérique, ainsi le 14 décembre, en évoquant " [le] général Rochambeau qui a eu tant de part au triomphe de la liberté américaine », et le 24 décembre, en rappelant « le souvenir des services rendus par le général Rochambeau à la cause de l'indépendance américaine » (Archives parlementaires de 1787 à 1860, op. cit., 1891, XXVI, p. 114 et 336).

(50) Le 24 décembre, Mathieu Dumas définit Luckner comme celui qui avait décidé de consacrer « à la France libre les talents qui firent souvent triompher nos ennemis, [et qui était] l'égal des grands capitaines de [son] siècle». 
du souverain restait faible. Il s'arrêtait en effet au stade de la proposition des noms, désormais soumise à l'examen du Comité militaire de l'Assemblée. Le député Delacroix voulut présenter la proposition de Narbonne comme inconstitutionnelle, en vertu de la loi du 4 mars $1791^{51}$. Dumas rappela alors le droit constitutionnel de Louis XVI de nommer des maréchaux, a fortiori quand il s'agissait d'une " mesure extraordinaire ». La discussion et la décision étaient désormais entre les mains du pouvoir législatif.

Le 24 décembre 1791, Mathieu Dumas présenta un projet de décret :

«L'Assemblée nationale, sur le vœu du roi, manifesté par le ministre de la Guerre, d'élever les lieutenants-généraux Rochambeau et Luckner, au grade de maréchal de France, considérant l'avantage qui en résultera pour le bien du service, et voulant donner à ces généraux, au moment où une grande partie des forces nationales leur est confiée, une preuve authentique de la confiance de la nation [...], décrète ce qui suit : Art. ${ }^{\text {er }}$ : Deux officiers généraux, commandants d'armée, pourront être élevés au grade de maréchal de France, sans que les places qu'ils occuperont puissent être considérées comme une augmentation permanente au nombre de six, auquel a été borné, par le décret du 4 mars dernier, celui des maréchaux de France en activité. Art. 2 : Lorsque, par la suite, il viendra à vaquer une place de maréchal de France, il ne pourra être pourvu au remplacement que conformément à la loi du 4 mars 1791, et sans que le nombre des maréchaux de France puisse excéder celui de six $»^{52}$.

Le texte paraît lui aussi conforme à la constitution, avec un roi des Français chef de l'exécutif, représenté par un ministre devant une Assemblée à l'écoute des vœux de l'exécutif. Cela n'était pourtant qu'une apparence, puisque le roi n'avait plus le pouvoir de décider du jour de promotion, comme cela se faisait jadis. Les discussions pour l'adoption du décret furent reportées au 27 décembre ${ }^{53}$. C'est ce jour-là que le décret fut voté, à la quasi-unanimité.

Le 28 eut lieu le dernier acte législatif. Cambon dénonça l'inconstitutionnalité du décret voté, qui n'exprimait pas, selon lui, une volonté

(51) Jean-François Delacroix, député à la Législative, guillotiné avec les Dantonistes le 5 avril 1794. Il ne faut pas le confondre avec le ministre des Relations extérieures du Directoire.

(52) Archives parlementaires de 1787 à 1860, op. cit., XXVI, p. 337.

(53) « Nous n'avons pas besoin tout à l'heure de deux maréchaux de France », affirma Delacroix. 
royale authentique. Dumas proposa alors une nouvelle formulation, à savoir : "L'Assemblée nationale, sur le vœu manifesté par le ministre de la Guerre et converti en motion par l'un de ses membres[...] $\gg^{54}$. Il désirait alors ôter la référence au roi, confirmant le divorce entre celui-ci et l'Assemblée. L'inconstitutionnalité de cette nouvelle rédaction fut là aussi soulignée par Delacroix, tandis qu'un autre député, Garran-de-Coulon, considérait que le décret était aussi voulu par Louis XVI. Le nouveau préambule fut finalement proposé par Cambon :

« L'Assemblée nationale, voulant faciliter au roi les moyens d'élever les généraux Luckner et Rochambeau au grade de maréchal de France, et voulant leur donner, au moment où une grande partie des forces de la Nation leur est confiée, une preuve authentique de la confiance de la Nation, décrète qu'il y a urgence $»^{55}$.

Ce texte fut adopté, et reproduit dans les journaux. Le Moniteur se contenta en revanche, dans le $n^{\circ} 363$ du 29 décembre, de rapporter le résultat des débats en précisant que «l'Assemblée [ordonna] la suppression dans le préambule de l'énonciation d'une proposition du roi ${ }^{56}$. Le texte final du décret n'avait pas effacé l'existence du roi, mais il avait donné l'initiative de la nomination des maréchaux à l'Assemblée, ce qui était visiblement inconstitutionnel. Curieusement, les Archives parlementaires ne s'achèvent pas au préambule de Cambon :

«Suit la teneur du préambule tel qu'il a été adopté lors de la lecture du procès-verbal : L'Assemblée nationale, voulant faciliter l'élévation des généraux Rochambeau et Luckner au grade de maréchal de France, et donner à ces généraux, au moment où une grande partie des forces nationales leur est confiée, une preuve authentique de la confiance de la Nation, décrète qu'il y a urgence $»^{57}$.

Cette variante du préambule, non diffusée, est étrange, car elle met en évidence la mise à l'écart du roi. Louis XVI, dont le crédit n'avait fait que décroître, avait totalement perdu l'initiative de nommer des maréchaux.

(54) Archives parlementaires de 1787 à 1860, op. cit., XXVI, p. 467.

(55) Ibid.

(56) Réimpression de l'Ancien Moniteur, op. cit., Paris, Bureau central, X, 1842, p. 747.

(57) Archives parlementaires de 1787 à 1860, op. cit., XXVI, 1887, p. 468. 


\section{L'impossible grade}

La cérémonie en faveur de Rochambeau et Luckner eut lieu à Metz le 2 janvier $1792^{58}$. Pour la première fois, ce n'était pas le roi, confiné aux Tuileries, qui donnait le bâton, mais Narbonne, qui prononça un discours soulignant les raisons pour lesquelles ils furent choisis comme maréchaux ${ }^{59}$. La rédaction de lettres patentes et d'une lettre aux promus toutes deux signées par le roi furent un moyen de préserver les apparences du pouvoir royal, même si le nouvel ordre était omniprésent ${ }^{60}$. Ces deux documents, ainsi que le bâton de maréchal, marquaient une continuité avec l'ancien ordre. Le bâton était l'élément le plus symbolique de l'amalgame entre l'Ancien Régime et les principes nouveaux du pays. En effet, il s'agissait du modèle de bâton défini en 1758, à un détail près ; les embouts n'étaient plus ornés d'une fleur de lys mais de la devise « LA NATION, LA LOI, LE ROI $\gg^{61}$. Tout fut mis en scène pour montrer que le grade

(58) Révolution française, ou Analyse complète et impartiale du Moniteur, Paris, Girardin, An IX (1801), I, p. 601.

(59) Ces raisons étaient analogues à celles évoquées par Mathieu Dumas : «Vous, M. de Rochambeau, qui avez courageusement combattu pour la liberté d'une partie du monde [...] Vous, M. de Luckner, que nous n'avions appris autrefois à connoître que par nos revers, vous nous avez adoptés pour patrie, et en privant nos ennemis d'un de leurs premiers généraux » (Discours adressé par M. de Narbonne, à MM. de Luckner et Rochambeau, au nom du Roi, à la tête de la garnison de Metz, 1792, p. 2).

(60) Rochambeau nota dans ses Mémoires que « le Roi signa de sa main [ses] lettres-patentes de maréchal de France » (Jean-Baptiste-Donatien de Vimeur DE Rochambeau, op. cit., I, p. 391). Sachant que les lettres patentes de maréchal étaient envoyées après la réception du mémoire des services rédigé par le promu, c'est-à-dire plusieurs semaines après la nomination, nous ne pensons pas qu'il les avait reçues. Le maréchal évoqua logiquement la lettre signée « Louis » et reçue en même temps que le bâton de maréchal à Metz. Les lettres patentes étaient toujours signées par le roi, y compris sur de nombreuses copies présentes dans différents fonds manuscrits. L'exemplaire de celles de Luckner, conservé dans son dossier militaire au SHD (2 Yd 255), ne se termine pas par la signature du roi. Est-ce un élément significatif? La lettre écrite par le roi aux deux nouveaux maréchaux a été placée à l'annexe 1 du présent article. Jusqu'à la Révolution, cette lettre était envoyée uniquement au promu, qui était le seul habilité à la décacheter. La lettre de Louis XVI eut la particularité d'avoir été diffusée dans la presse. À l'instar des débats à l'Assemblée sur la nomination exceptionnelle de deux maréchaux supplémentaires, la lettre au promu était un signe du transfert du système de nomination de la sphère privée (entre le roi et le nouveau maréchal) à la sphère publique (débats dans une assemblée, publication de ceux-ci, publication de la lettre adressée au nouveau titulaire). En ce qui concerne l'omniprésence du nouvel ordre dans les lettres patentes délivrées, nous renvoyons à Gabriel Le BARROIS D'Orgeval, op. cit., II, p. 51. L'auteur démontre avec justesse ce fait en analysant le brevet de Luckner.

(61) Plusieurs clichés du bâton de Luckner, conservé au Musée de l'Armée, ont été réalisés et publiés sur le site de la Réunion des musées nationaux (http://www.photo.rmn.fr ; nous tenons à remercier Messieurs Dominique Prévôt et Laurent Bergeot qui ont facilité la prise des clichés et leur mise en ligne, suite à nos demandes de renseignements sur ce bâton notamment). Une description très précise de cet objet est donnée dans l'article de M. D. MAC CARThy, « Le Bâton du maréchal de 
de maréchal de France était compatible avec la Révolution, et qu'il fournissait un potentiel de généraux de qualité.

Les espoirs portés sur Rochambeau et Luckner furent pourtant rapidement déçus. La désorganisation de l'armée, en plus du fait qu'ils incarnaient une guerre d'un autre temps, les empêcha d'agir de façon satisfaisante. Rochambeau, constatant lui-même qu'il ne contrôlait pas la situation de l'armée du Nord, manifesta dans une lettre lue à l'Assemblée législative le 4 mai 1792 son désir de quitter son commandement. Deux députés crièrent alors des phrases comme "Qu'il renvoie le bâton! » et «Le renvoi à Orléans! $»^{62}$. Fidèles au retour de l'idée de l'obtention du bâton aux commandants d'armée, et non pas à celle de la récompense d'une carrière, ces deux députés restés anonymes désiraient finalement le retour à un principe disparu au $\mathrm{XVI}^{\mathrm{e}}$ siècle. À l'époque, le roi pouvait ôter à un maréchal sa charge si celui-ci était disgracié, promu à une autre charge, ou retiré de l'armée. Seulement, en 1792, ce n'était pas le souverain qui émettait un tel désir. Cette réclamation fut sans suite, mais elle montrait que l'existence du maréchalat régénéré était précaire.

Luckner resta à l'armée un peu plus longtemps, même s'il était évident que ses qualités militaires réelles faisaient « qu'il n'avait jamais été et qu'il ne serait jamais autre chose qu'un colonel de hussards $»^{63}$. Passé de l'armée du Rhin à celle du Nord, en remplacement de Rochambeau, il pénétra en Belgique, mais battit peu après en retraite. Voulant lui accorder encore sa confiance, l'Assemblée le nomma à l'armée du Centre, puis au camp de Châlons en tant que généralissime, à la demande de Kellermann. Il était officiellement chargé « d'aider de ses conseils les généraux des différentes armées; [de...] former dans cette ville une réserve de troupes propre à recevoir les débris des armées battues $»^{64}$. Luckner devait donc s'occuper des camps et des armées, ce qui rapprochait sa tâche du rôle originel du maréchal-général des camps et armées du roi, en même temps qu'elle lui conférait un statut supérieur, quoique

(suite de la note 61 page 66)

Luckner ", Revue de la SAMA, 1968, n 72, p. 29-30. Le bâton présenté comme étant celui de Rochambeau, exposé le 8 septembre 2007 au château de Rochambeau, ne peut en revanche être considéré comme un objet authentique.

(62) Archives parlementaires de 1787 à 1860, op. cit., XLII, 1893, p. 740. Voulait-on le donner à Philippe-Égalité, ou alors à son fils, le futur Louis-Philippe, qui se distingua plus tard à Jemmapes ? Le problème est que l'un ne commandait pas et que l'autre n'avait que dix-neuf ans...

(63) Vincent-Marie-Viennot de Vaublanc, Mémoires de M. le comte de Vaublanc, Paris, Firmin Didot, 1857, I, p. 204.

(64) Antoine-François Bertrand de Molleville, Histoire de la Révolution de France, deuxième partie, Paris, Giguet et Michaud, 1802, IX, p. 226. 
simplement honorifique. Sa principale action fut l'impression d'un placard signé de sa main appelant les soldats à rentrer chez eux. Cela le rendit naturellement suspect auprès du commissaire du Conseil exécutif Choderlos de Laclos, qui le dénonça dès le 10 septembre au ministre Servan ${ }^{65}$.

Entre-temps eut lieu le 10 Août. Le 20 septembre fut remportée la bataille de Valmy, et le lendemain fut proclamée la République. Le 22, la Convention, nouvelle Assemblée, convoqua Luckner à Paris pour s'expliquer sur sa retraite en Belgique. Plus aucun maréchal de France n'était à l'armée. Cette situation fut remarquée par le vainqueur de Valmy, Kellermann. Le 21 octobre 1792, après avoir repris Longwy, celui-ci, fort de ses succès, écrivit à Fabre d'Églantine une lettre dans laquelle il faisait remarquer que Dumouriez, Biron, Montesquiou, et lui-même étaient des généraux en chef, " grade [...] intermédiaire de celui de général et de maréchal $»^{66}$. Il demandait pour ses pairs ainsi que pour lui « le grade de maréchal de la République », suggérant même de le conférer à Custine. Une semaine après, le général Anselme prit Nice. Une « lettre des corps administratifs réunis de la ville et du ci-devant comté de Nice » fut apportée à la Convention. Elle demandait à ce qu'on conférât à Anselme « le grade de maréchal de France $»^{67}$. Les réactions à la Convention furent très négatives. Le député Gensonné affirma que le maréchalat était « un titre qui [devait] être aboli ». Quant au député Lasource, il fut plus explicite en réclamant "l'anéantissement du titre de maréchal de France », suscitant des applaudissements ${ }^{68}$.

Ces déclarations fortes et spontanées sont à rapprocher des propos tenus le 27 décembre 1791 par un député dont le nom ne fut pas retenu par les Archives parlementaires. Celui-ci avait dit « que des serviteurs de la patrie n'ont pas besoin de recevoir des honneurs pour la bien servir $»^{69}$. Il est évident que les marques d'honneur étaient alors appelées à disparaître, car elles étaient des vestiges du " monde "féodal" » d'après Jean-Paul Bertaud. De telles distinctions ne faisaient qu'établir des inégalités entre

(65) Jean-Paul Bertaud, Choderlos de Laclos, Paris, Fayard, 2003, p. 385.

(66) Jean-François-Eugène RoBinet, Le Procès des Dantonistes, Paris, Leroux, 1879, p. 531 .

(67) Archives parlementaires de 1787 à 1860, op. cit., 1898, LIII, p. 25.

(68) Ibid.

(69) Archives parlementaires de 1787 à 1860, op. cit., XXVI, p. 462. 
les citoyens, tous appelés à servir la patrie avec un esprit de renoncement et de vertu ${ }^{70}$.

Cette évolution des conceptions de l'honneur contribua à l'abolition du maréchalat, qui ne fut toutefois décidée que le 21 février 1793. Son non-remplacement par un grade équivalent soulignait l'idée qu'il incarnait également un objet d'ambition dangereux pour la République ${ }^{71}$.

\section{Les « ex-maréchaux »}

Il restait dans la République française, outre Broglie et Castries en exil, des " ci-devant maréchaux de France », appelés aussi " ex-maréchaux ». La rupture ne fut pas radicale, car le 26 avril 1793, Bouchotte, alors ministre de la Guerre, posa la question du paiement du traitement des anciens maréchaux de France d'active, à la suite de courriers émanant des intéressés. L'affaire n'eut pas de suite.

La Terreur parut changer la donne, car elle vit l'exécution des maréchaux de Mailly, de Mouchy, et Luckner. Rochambeau échappa de peu à la guillotine, tandis que le nonagénaire Contades fut juste assigné à résidence. Ce n'était pas le maréchalat qui était visé, car le maréchal de Laval mourut en 1798 sans avoir été inquiété. C'étaient leur noblesse, le département où ils se trouvaient, et leurs activités qui étaient susceptibles de leur causer des ennuis. Le maréchal de Mouchy fut guillotiné pour avoir hébergé des prêtres réfractaires. Quant à Mailly, son arrestation fut décidée à cause du zèle du représentant en mission dans la Somme, Dumont, comme en témoigne une lettre de ce représentant à la Convention datant des environs du 7 septembre 1793 :

« J'ai à peine le temps de vous écrire; je crois que tous les ci-devant ducs, comtes, vicomtes, marquis, etc., et leurs familles sont dans ce pays [...] Dans les nouvelles arrestations, les Mailly, les Beuvron, les d'Harcourt, les de Ligne s'y trouvent compris. Les titres de noblesse sont saisis $»^{72}$.

(70) Jean-Paul Bertaud, Quand les enfants parlaient de gloire, Paris, Aubier, Collection historique, 2006, p. 169.

(71) Les rumeurs d'intrigues menées par certains commandants pour obtenir le grade de maréchal de la République paraissent relativement fréquentes à la fin de l'année 1792, car, hormis les exemples que nous avons relevés, nous pouvons aussi mentionner l'évocation, dans une lettre de Roland à Pache qui daterait de la fin du mois de décembre, d'un commandant démissionnaire dont le «but est d'être proclamé par le peuple, maréchal de la République » (Le Procès Pache, Paris, Cornely, 1911, p. 22).

(72) Recueil des actes du Comité de salut public, Paris, Imprimerie nationale, Collection des documents inédits sur l'histoire de France, 1893, VI, p. 333. 
C'est pour les mêmes causes que la dépouille de Fabert disparut, lui qui apparut comme un homme du tiers état aussi bien avant que pendant la Révolution, et évidemment après. Les " maréchaux roturiers » furent récupérés en fait par les royalistes, afin de montrer que les bourgeois avaient pu accéder aux plus hautes dignités sous l'Ancien Régime. En 1795, Sénac de Meilhan publia son ouvrage Du gouvernement, des mours et des conditions en France, avant la Révolution, dans lequel est présentée une liste de maréchaux prétendument issus de la bourgeoisie ${ }^{73}$. Elle comporte curieusement les noms de Belle-Isle, d'un maréchal dont le nom est dissimulé ( « le Maréchal de *** », probablement Broglie), de Villars, de Maillebois, de Fabert, de Catinat et d'Asfeld. Le propos restait le même, mais il passa pour ainsi dire dans le camp opposé.

Les exécutions de Mailly et de Mouchy mirent en évidence le fait que les maréchaux étaient avant tout fidèles au roi et à la religion catholique. Mouchy était présent aux Tuileries le 20 juin 1792, tandis que Mailly l'était le 10 Août suivant. Le second participa à la défense du château contre les Fédérés, malgré ses quatre-vingt-quatre ans, mais son titre de maréchal le sauva d'une mort certaine par deux fois, le 10 août et le 2 septembre ${ }^{74}$. En montant à l'échafaud à Arras, Mailly cria « Vive le roi ! Je le dis comme mes ancêtres ! $\gg^{75}$. À Paris, Mouchy aurait affirmé : « À dix-sept ans, j'ai monté à l'assaut pour mon roi ; à soixante-dix-huit, je vais à l'échafaud pour mon Dieu $\rangle^{76}$.

L'exécution de Luckner, et celle de Rochambeau qui n'eut jamais lieu, sont plus à rapprocher des exécutions de généraux de la République qui battirent en retraite, comme Beauharnais ou Custine. Cela est simplement dû au fait qu'ils furent les seuls maréchaux de France à avoir commandé une armée sous la Révolution.

Paradoxalement, même durant la Terreur, il arrivait que le titre de maréchal de France ne fût pas présenté comme un titre révolu. Certes, Le Moniteur universel, en annonçant l'exécution du maréchal de Mouchy, désigna celui-ci comme « ex-duc, ex-maréchal de France, ex-gouverneur de Versailles », mais le même journal était plus ambigu concernant Beauvau, en mentionnant la mort du « ci-devant prince de Beauvau, maréchal de

(73) Gabriel Senac de Meilhan, Du Gouvernement, des mours, et des conditions en France, avant la Révolution, Hambourg, Hoffmann, 1795, p. 66. III, p. 486.

(74) Charles-Élie DE Ferrières, Mémoires du marquis de Ferrières, Paris, Baudouin, 1821,

(75) Ibid.

(76) Louise-Henriette dE DuRAs, Journal des prisons de mon père, de ma mère et des miennes, Paris, Plon, 1888, p. 285. 
France », quoique le terme " ci-devant » s'appliqua sûrement aux deux titres du défunt ${ }^{77}$. Que penser de l'anecdote sur Rochambeau, qui évita de justesse la guillotine ? Alors qu'il devait être exécuté, on appela les personnes destinées à monter dans la charrette. Rochambeau, d'après ses Mémoires, s'apprêta à avancer, quand on lui cria qu'il ne faisait pas partie du nombre : «Tu n'as donc pas entendu, maréchal [...] ? Il n'y a rien pour to $\gg^{78}$. Une autre version, plus connue, a un aspect plus brutal. Le maréchal faisait partie des condamnés devant partir pour l'échafaud, mais le bourreau se rendit compte que la charrette était trop lourde. Il aurait repoussé Rochambeau en lui affirmant "Retire-toi, vieux maréchal; ton tour viendra plus tard ». Quelle que soit la variante que l'on adopte, Rochambeau est toujours appelé de la même manière : « Maréchal ».

À l'instar de la Convention, le Directoire ne rétablit pas le grade de maréchal de France, pas même en faveur des nombreux brillants généraux qui avaient permis de faire de la France l'arbitre de l'Europe. Le rétablissement d'une dignité d'Ancien Régime n'avait pas sa place dans un régime républicain.

\section{4 : le rétablissement de la dignité de maréchal de France?}

Quand Bonaparte devint Premier Consul, l'une de ses premières tâches fut de réconcilier les deux France : «ni talons rouges, ni bonnets rouges ». Époux d'une veuve de général guillotiné, il avait conscience qu'il fallait réhabiliter les généraux disparus, ainsi que ceux qui avaient survécu. Les maréchaux nommés sous la Révolution furent parmi les premiers dont l'action fut réévaluée, dont la stature fut reconsidérée. L'anecdote de Bonaparte rendant visite à Rochambeau avec Berthier, ancien aide-de-camp de ce dernier en Amérique, est bien connue ${ }^{79}$. Il est à noter que le Consul l'appela " général », ce qui est après tout logique, car le grade de général de division était désormais le plus élevé de la hiérarchie militaire. C'est aussi pour cela qu'il reçut, de même que le «ci-devant maréchal de Ségur », une pension de général de division réformé ${ }^{80}$.

Malgré ses erreurs, Luckner fut également perçu d'une autre façon. Une estampe datant de l'an VIII le représente, ainsi que son arrestation ${ }^{81}$.

(77) Réimpression du Moniteur, op. cit., XXI, p. 112, et XVI, p. 473.

(78) Jean-Baptiste-Donatien de Vimeur de Rochambeau, op. cit., II, p. 40.

(79) Le Premier Consul dit à Rochambeau : « Général, voilà vos élèves ». Rochambeau répondit : « Les élèves ont bien surpassé leur maître ».

(80) Elle s'élevait à six mille francs. «Le citoyen Philippe-Henry Ségur, ci-devant maréchal de France et ministre de la guerre, jouira des appointements de général de division réformé » (SHD, dossier 2 Yd 250).

(81) Cette estampe se trouve au château de Versailles. 
Un texte, intitulé "Luckner généralissime des armées françaises, en 1792 », se situe au-dessous. Il réhabilite complètement celui-ci, « qui, dans le court commandement qu'il eut pendant la guerre de la Révolution n'avoit aucun reproche à se faire, fut envoyé à l'échaffaud, lors du regne de la terreur, par ce tribunal de sang ». Le texte se conclut ainsi : " Ainsi périt ce respectable vieillard, qui avoit cru trouver une retraite paisible parmi nous. mais qu'elle [sic] terre pouvoit être hospitalière, sous un tigre tel que Robespierre!»

En même temps étaient officiellement et pompeusement célébrés les grands militaires de l'Ancien Régime, tels Turenne, dont le corps fut déposé aux Invalides en 1800, et plus tard Vauban, dont le cœur y fut aussi placé en $1808^{82}$.

L'État napoléonien naissant remit en valeur la culture de l'honneur, et exalta les généraux victorieux. Ce nouveau régime avait besoin de fidéliser ceux-ci, républicains endurcis ou anciens serviteurs de l'Ancien Régime. Il était évident que les serviteurs de la patrie avaient besoin de recevoir des honneurs pour bien servir Bonaparte, devenu l'empereur à qui était confié le gouvernement de la République.

Aussi est-ce pour cela que l'article 48 de la nouvelle constitution de l'an XII instituait des grands officiers de l'Empire. Les premiers étaient les maréchaux d'Empire, " choisis parmi les généraux les plus distingués », qui ne devaient pas être plus de seize, exclusion faite des maréchaux sénateurs. Rochambeau ne faisait pas partie du nombre, mais il avait une pension d' « ancien maréchal de France », titulature que nous retrouvons en frontispice de ses mémoires. Contrairement au grade qu'il avait reçu avec Luckner, le titre de maréchal d'Empire était une dignité civile, mais cela n'empêchait pas les attributions de fonctions militaires, bien au contraire.

La référence à la loi constitutionnelle dans les patentes de maréchal rappelle ce qu'on pouvait trouver dans le brevet de Luckner en $1791^{83}$. Si la liste des promus fit la part belle aux généraux issus des guerres de la Révolution, elle incluait aussi des officiers ayant servi dès l'Ancien Régime, tel Kellermann, qui obtenait enfin ce qu'il avait revendiqué en 1792. La dignité « rétablie » par Napoléon n'avait pas non plus grand-

(82) Sur les péripéties subies par la dépouille de Turenne entre 1793 et 1800, ainsi que sur le déroulement et le sens de la cérémonie des Invalides, nous renvoyons au remarquable article de Bronislaw BACZKo, « Turenne au temple de Mars », dans Jean-François Fayet, Carine Fluckiger et Michel Porret [dir.], Guerres et paix : mélanges offerts à Jean-Claude Favez, Genève, Goerg, 2000, p. $75-89$.

(83) Nous pouvons le constater avec les lettres-patentes d'Augereau, citées par Gabriel LE Barrois D'Orgeval, op. cit., II, p. 488. 
chose à voir avec celle de l'Ancien Régime. Les institutions comme le tribunal n'avaient pas été rétablies, de même que le profil des titulaires était sensiblement différent. Hormis Kellermann et Sérurier, âgés respectivement de soixante-neuf et soixante-deux ans, ils étaient bien plus jeunes que la plupart des maréchaux nommés depuis Louis XV.

Seules la fournée de dix-huit maréchaux en 1804 et la place qu'ils tinrent lors de la cérémonie du sacre pouvaient réellement rappeler l'Ancien Régime. On peut admettre que la fournée fut nécessaire, dans la mesure où il fallait constituer les effectifs de la dignité nouvellement créée. En revanche, il est indéniable que le rôle des maréchaux dans le sacre était directement inspiré de celui qui était le leur en 1775 pour celui de Louis XVI. Kellermann, Pérignon, Lefebvre, Bernadotte et Berthier portèrent les honneurs en 1804 au même titre que Contades, Broglie et Nicolaÿ en $1775^{84}$.

Ce que la République ne pouvait se permettre de faire pour des raisons idéologiques et politiques, l'Empire le fit sans difficulté. La création du maréchalat d'empire répondait à un changement du système militaire. Les généraux en chef n'existaient plus. Napoléon était le commandant, le tacticien et le stratège suprême. L'analogie entre la conduite des opérations par l'empereur et la stratégie de cabinet de l'ère louisquatorzienne peut être séduisante, car les maréchaux qui servaient à l'armée étaient appelés à n'être que des exécutants. Toutefois, il convient de rappeler que Napoléon ne dirigeait pas ses armées depuis les Tuileries, comme Louis XIV essayait de le faire à Versailles. À de rares exceptions près (Davout, Lannes), les maréchaux d'Empire furent de bons exécutants, de bons lieutenants. À l'opposé des règnes de Louis XIII et de Louis XIV, celui de Napoléon n'était pas marqué par la recherche et la promotion de grands capitaines, car l'empereur était censé être l'unique grand capitaine. Le XVII siècle fut en France le siècle des Turenne, Condé et Luxembourg. Le XVIII fut celui des Vendôme, Villars et Saxe. La Révolution fut la décennie des généraux en chef, dans la lignée des grands chefs des deux siècles précédents, avec Hoche et Bonaparte notamment. Le XIX ${ }^{\mathrm{e}}$ siècle fut indéniablement le siècle de Napoléon, même si, sur le plan purement quantitatif, il ne représenta que quinze ans de ce siècle, quinze ans durant lesquels on ne vit jamais autant de maréchaux désignés.

(84) Procès-verbal de la cérémonie du sacre et du couronnement de LL. MM. l'empereur Napoléon et l'impératrice Joséphine, Paris, Imprimerie impériale, 1805, p. 23 ; Journal historique du sacre et du couronnement de Louis XVI, roi de France et de Navarre, 1775, p. 72. 
Le maréchalat survécut sans difficulté à l'Empire. Louis XVIII permit un habile mélange entre les héritages de l'Ancien Régime et de l'Empire, mais il faut avouer que les différences n'étaient pas toujours très fortes, en particulier au niveau du rôle des maréchaux lors du sacre. D'ailleurs, le serment de maréchal en vigueur sous l'Ancien Régime réapparut, à quelques mots près, de même que le bâton d'origine, après ceux semés d'aigles d'or. La monarchie de Juillet reprit quant à elle l'idée de limitation des effectifs en temps de paix telle qu'elle fut débattue en 1791, mais la véritable remise à plat de l'attribution et des fonctions de cette dignité n'eut lieu que suite à la longue interruption de son attribution entre 1870 et 1916.

Fadi El HaGe

5 rue Carnot appartement 101

93000 Bobigny elhage.fadi@wanadoo.fr 


\section{ANNEXE}

Lettre du roi envoyée aux maréchaux de Rochambeau et Luckner, datée du 28 décembre 1791 (B.C. GournaY, Journal militaire contenant tout ce qui est relatif à l'Organisation, à la Composition et à l'Administration de la FORCE PUBLIQUE ; et enfin tout ce qui concerne la guerre. Troisième année. Première partie, Paris, Firmin Didot, 1792, p. 38).

«Paris, le 28 décembre 1791.

L'Assemblée nationale a secondé mes désirs, Monsieur, en me mettant à portée de vous donner une marque éclatante de satisfaction et d'estime.

La dignité à laquelle je vous élève, en même temps qu'elle est la récompense de vos services passés, doit être pour vous un puissant motif d'en rendre de nouveaux à la patrie, et de répondre à l'attente de la Nation et à ma confiance.

Employez tous vos soins, Monsieur, à rétablir la discipline militaire, elle est le gage assuré du succès pendant la guerre, ce qui est plus précieux encore, elle est souvent un moyen de l'éviter.

Signé, LOUIS ». 
